\title{
RANDOM SPHERES AND OPERADS
}

\section{RÉMI LÉANDRE}

Communicated by Martin Schlichenmaier

\begin{abstract}
We define a stochastic diffeology over the $n$-fold loop-space of a $n$ connected manifold. We perform a stochastic cohomology in the sense of Chen and Souriau and we show that the stochastic cohomology groups are equal to the classical de Rham cohomology groups of the $n$-fold loop space. We give a stochastic analogue of the classical fact that the homology groups of the $n$-fold loop space are an algebra for the little cube operad.
\end{abstract}

\section{Introduction}

In algebraic topology (see [8], [13], [16], [40]), people consider as models on the $n$-fold loop space $\Omega_{x}^{n}(M)$, the space of maps from $\mathbb{R}^{n}$ into the compact manifold $M$ which are equal to $x$ outside a cube (which can depends on the map). The $n$ fold loop space is not the same as the set of applications from the $n$-dimensional sphere $\mathbb{S}^{n}$ to $M$ which sends the north pole of the sphere to $x$. The interest of the $n$-fold loop space is that there are many different structures. For instance, it carries an action of the little cube operad [40], such that its homology appears as an algebra associated to this operad or in a more sophisticated way, as a $n$-algebra [13].

The goal of this article is to define a stochastic $n$-fold loop space and to define a stochastic homology theory which is compatible with the action of the little cube operad.

There is something analogous in one dimension: the Moore loop space. Taking into consideration as a model of the loop space, the based loop space endowed with Brownian bridge measure, a tentative description of the stochastic Moore loop space was produced in [21] which is involved with the long time behavior of the heat kernel. The cohomology of the smooth Moore loop space is an Hopf algebra, but our program in [21] fails in the definition of suitable tensor products of Sobolev forms if we consider Sobolev cohomology of the stochastic Moore loop space. 
There are two types of cohomology theory for the Brownian bridge, which deal with forms almost surely defined:

The first one is related to Sobolev cohomology theory. To a random form, which is almost surely defined, is associated a series of Sobolev norms, as is classical in Malliavin Calculus. Namely the main originality of Malliavin Calculus with respect of its precursors (see works of Albeverio, Elworthy, Hida, Fomin, Berezanskii...) is that the space of test functions is an algebra constituted of functional, almost surely defined, because there is no Sobolev imbedding theorem in infinite dimension. The main theorem, which gives a stochastic extension of the classical results in the algebraic topology of Chen and Adams, is the following: if the manifold is simply connected, the Sobolev cohomology of the Brownian bridge is equal to the Hochschild cohomology ([18], [22], [23], [29]). Therefore, the stochastic cohomology in the Sobolev sense of the loop space is equal to the cohomology of the smooth loop space.

The second one is related to the stochastic calculus of Chen and Souriau, which is a stochastic interpretation of the considerations of Chen and Souriau for diffeology ([12], [17], [45]). We will not discuss here the Hilbert tangent space over a random loop, which was introduced by Jones and Léandre [18] and by Bismut in a preliminary form [7]. Therefore, a Sobolev norm is not associated with a random form. The stochastic cohomology for much diffeology is equal to the cohomology of the smooth loop space, or to the cohomology of the Hölder loop space ([27], [28], [30]).

In the first part of this article, we will construct a stochastic diffeology over $\Omega_{x}^{n}(M)$, our considerations are related to the construction of the heat kernel measure over a loop group of Airault and Malliavin. We consider some stochastic processes over $\Omega_{x}^{n}(M)$, which depend continuously on a finite-dimensional parameter. Our construction of some examples of random plots with values in the $n$-fold loop space are, in fact, $n+1$-dimensional, and are inspired by the construction of Léandre [34] and [36] in order to construct random tori and random cylinders, and not by the construction of Airault and Malliavin [1], Brzezniak and Elworthy [10], Brzezniak and Léandre [11] and Léandre [35] in order to construct random cylinders, where the models were two dimensional.

In Section 2, starting from the heat kernel diffeology, we construct a stochastic Chen-Souriau diffeological Calculus of the $n$-fold loop space. The main theorem is that the stochastic cohomology with respect of this diffeology of the stochastic $n$-fold loop space, is equal to the cohomology of the Hölder $n$-fold loop space. The proof is not based upon the sheaf cohomology, as it was done in [28] for loop space and in [35] for tori, but is an analogy of the proof of [30] based upon the 
Cech cohomology associated with some cover of the loop space (see [9] pp. 166167 for the abstract statement of this proof).

In Section 3, we define an action of the little cube operad over the stochastic $n$-fold loop space and we deduce that the stochastic homology groups of the $n$-fold loop space are an algebra with respect to this operad. This is possible because we take a very rich stochastic diffeology and therefore a rather poor class of functionals, unlike the poor diffeology of [34] which is not compatible with the action of the little cube operad, but which is involved with a much more larger class of functionals.

This article requests the study of diffusion processes over infinite-dimensional manifolds. In a abstract way, these processes were studied by Kuo [19] and the russian school [5]. But our work is motivated by the higher-dimensional considerations of the works of Airault and Malliavin [1] and Brzezniak and Elworthy [10].

We refer to the two surveys of Léandre ([26], [32]) for analysis over loop space and topology.

\section{Stochastic $n$-fold Loop Space}

Let us consider the Hilbert space of functions $f$ from $\mathbb{R}$ into $\mathbb{R}$ such that

$$
\int_{\mathbb{R}}|f(s)|^{2} \mathrm{~d} s+\int_{\mathbb{R}}\left|f^{\prime}(s)\right|^{2} \mathrm{~d} s=\langle f, f\rangle<\infty .
$$

The map which associates $f(t)$ to $f$ is continuous for the Hilbert structure. Therefore there exists an element $e_{t}\left(t^{\prime}\right)$ from the Hilbert space such that $f(t)=\left\langle f, e_{t}\right\rangle$. This element $e_{t}$ is called the Green kernel associated to the Hilbert space. We can explicitely compute $e_{0}$. It is equal to $\lambda 1_{t^{\prime} \leq 0} \exp \left[t^{\prime}\right]+\mu 1_{t^{\prime}>0} \exp \left[-t^{\prime}\right]$ (for some convenient choice of $\lambda$ and $\mu$ ) and $e_{t}$ is deduced by translation. To the Hilbert space is associated an operator $I-\partial^{2} / \partial s^{2}$. We consider the Hilbert by using the functions from $\mathbb{R}^{n}$ into $\mathbb{R}$ associated to the product of operators $A=\prod_{i=1}^{n}\left(I-\partial^{2} / \partial s_{i}^{2}\right)$. We consider also the Hilbert space $\mathbb{H}$ of functions from $\mathbb{R}^{n}$ into $\mathbb{R}$ such that

$$
\int_{\mathbb{R}^{n}} h(S) A h(S) \mathrm{d} S<\infty .
$$

Since we consider a product situation, the Green kernel associated to this operator is $\prod e_{s_{i}}\left(s_{i}^{\prime}\right)=e_{S}\left(S^{\prime}\right)$ where $S=\left(s_{1}, . ., s_{n}\right)$. We consider a compact manifold, 
imbedded isometrically into $\mathbb{R}^{d}$, and we consider the set of functions from $\mathbb{R}^{n}$ into $\mathbb{R}^{d}$. We can explicitly compute the Green kernel (or the reproducing kernel), and obtain a Hilbert space $\mathbb{H}^{d}$ built by functions from $\mathbb{R}^{n}$ into $\mathbb{R}^{d}: S \rightarrow h(S)$ if $S=\left(s_{1}, . ., s_{n}\right)$. The base point $x$ of $M$ is identified with 0 in $\mathbb{R}^{d}$. In the sequel, we will consider the Brownian motion with values in $H: u \rightarrow B_{u}($.$) . This gives a$ $n+1$ parameter process. In this case $t \rightarrow B_{t}(S)$ is a finite dimensional Brownian motion and the correlation between $B .(S)$ and $B .\left(S^{\prime}\right)$ is given by $\left\langle e_{S}, e_{S^{\prime}}\right\rangle \operatorname{Id}_{\mathbb{R}^{d}}$ In the sequel, we will define by $\left(\Omega, F_{t}, P\right)$ the filtered probability space where this Brownian motion is defined.

In order to construct precisely the Brownian motion with values in $\mathbb{H}$, we consider an orthonormal basis $f_{i}$ of $\mathbb{H}$ and some independent $\mathbb{R}$-valued Brownian motion $B_{i, t} . B_{t}(s)$ is therefore equal to $\sum B_{i, t} f_{i}(S)$. We remark that with the previous definition we have

$$
\left\langle B_{t}(S), B_{t}\left(S^{\prime}\right)\right\rangle=t \sum f_{i}(S) f_{i}\left(S^{\prime}\right)=t\left\langle e_{S}, e_{S^{\prime}}\right\rangle .
$$

(We have as if the Gaussian field takes its values in $\mathbb{R}$ in order to simplify the exposition). $(t, S) \rightarrow B_{t}(S)$ is in fact only Hölder!

Let us recall that a continuous process $t \rightarrow M_{t}$ is a $\mathbb{R}$-valued martingale in $L^{2}$ with respect of the filtration $F_{t}$ if $E\left[\left|M_{t}\right|^{2}\right]<\infty$ and if for $t^{\prime}>t$

$$
E\left[M_{t^{\prime}} \mid F_{t}\right]=M_{t}
$$

almost surely. A semi-martingale is defined as the sum of a continuous martingale and of continuous finite variation process $t \rightarrow V_{t}$ such that $V_{t}$ is $F_{t}$-measurable for all $t$. The right-bracket of continuous martingale $M_{t}$ is the unique continuous increasing process $\langle M, M\rangle$ which is $F_{t}$ measurable and such that

$$
M_{t}^{2}-\langle M, M\rangle_{t}
$$

is a continuous martingale. The interest of the right-bracket of a martingales is the following: let $p>0$. The $L^{p}$ norm of $\sup _{t<1}\left|M_{t}\right|$ can be estimated in term of the $L^{p}$ norm of $\langle M, M\rangle_{1}^{1 / 2}$. It is the purpose of Burkholder-Davies-Gundy inequalities.

We consider a set of semi-martingales parametrized by $t \in[0,1]$ and depending of the parameter $S \in \mathbb{R}^{n}$

$$
X_{t}(S)=M_{t}(S)+V_{t}(S), \quad X_{0}(S)=x .
$$

We define over this set of semi-martingales a set of norms

$$
E\left[\left\langle M_{1}(S)-M_{1}\left(S^{\prime}\right), M_{1}(S)-M_{1}\left(S^{\prime}\right)\right\rangle^{p / 2}\right]^{1 / p} \leq C_{p}^{1} d\left(S, S^{\prime}\right)^{1 / 2}
$$


where $\langle$,$\rangle denotes the right bracket of semi-martingale theory and d\left(S, S^{\prime}\right)$ is the Euclidean distance over $\mathbb{R}^{n}$ for $d\left(S, S^{\prime}\right) \leq 1$. Moreover

$$
E\left[\left(\int_{0}^{1} \mathrm{~d}\left|V_{t}(S)-V_{t}\left(S^{\prime}\right)\right|\right)^{p}\right]^{1 / p} \leq C_{p}^{2} d\left(S, S^{\prime}\right)^{1 / 2} .
$$

We suppose that

$$
\begin{gathered}
E\left[\left\langle M_{1}(0), M_{1}(0)\right\rangle^{p / 2}\right]^{1 / 2} \leq C_{p}^{3} \\
E\left[\left(\int_{0}^{1} \mathrm{~d}\left|V_{t}(0)\right|\right)^{p}\right]^{1 / p} \leq C_{p}^{4} .
\end{gathered}
$$

Using Kolmogorov's lemma (see [41]), we deduce that the random field $S \rightarrow$ $X_{1}(S)$ is almost surely Hölder with an Hölder exponent $<1 / 2$. The random field is in fact almost surely $1 / 2-\epsilon$-Hölder for all $\epsilon>0$.

Definition 1. The space $S P_{\infty}$ is the space of semi-martingales $X$.(.) with values in $\mathbb{R}^{d}(2.2)$ submitted to the conditions that $C_{p}^{1}, C_{p}^{2}, C_{p}^{3}$ and $C_{p}^{4}$ are finite for all p. This endows $S P_{\infty}$ with a structure of Fréchet space.

Since $M$ is isometrically imbedded into $\mathbb{R}^{d}$, we consider the projection map $\Pi(y)$ from $\mathbb{R}^{d}$ into $T_{y}(M)$, the tangent space of $M$ at $y$. We consider the family of stochastic differential equations in the sense of Stratonovitch

$$
\mathrm{d}_{t} x_{t}(S)=g(S) \Pi\left(x_{t}(S)\right) \mathrm{d}_{t} B_{t}(S), \quad x_{0}(S)=x .
$$

Theorem 2. $x$.(.) belongs to $S P_{\infty}$. Moreover, $x_{1}(S)$ belongs to $M$ and is equal to $x$ outside a deterministic compact set of $\mathbb{R}^{n}$.

Proof: The proof is straightforward and based upon the Gronwall lemma and the continuity properties of the Green kernel. We refer to [36] for details.

We will define on the Hölder $n$-fold loop space $\Omega_{x, 1 / 2-\epsilon}^{n}(M)$ a stochastic diffeology $(1 / 2-\epsilon$ is the Hölder exponent). Let us explain why we fix the rate $1 / 2-\epsilon$ : the $1 / 2-\epsilon$ Hölder $n$-fold loop space $\Omega_{x, 1 / 2-\epsilon}^{n}(M)$ is an inductive limit of Banach manifolds, the injection map being the injection of maps equals to $x$ outside the cube $[-r, r]^{n}$ into the maps equal to $x$ outside the cube $[-(r+1), r+1]^{n}$. Maps equals to $x$ outside the cube $[-r, r]^{n}$ are said to belong to $\Omega_{x, 1 / 2-\epsilon}^{r, n}(M)$ which is a Banach manifold (see beginning of Section 3 for details). A functional on $\Omega_{x, 1 / 2-\epsilon}(M)$ is said Frechet smooth if its restriction to all $\Omega_{x, 1 / 2-\epsilon}^{r, n}(M)$ is 
Frechet smooth. Frechet differentiability on Banach manifolds is a tractable notion. If we consider the space of Hölder $n$-fold loops without to precise the rate of Hölderianity, the topological considerations are much more complicated. We should precise in this last case for instance what is a smooth functional on the Hölder $n$-fold loop space, which should lead to boring technical considerations.

Let us recall what is a diffeology following the terminology of Souriau: Let us consider a topological space $\tilde{M}$. A diffeology is constituted of a collection of maps $\left(\phi_{U}, U\right)$ from any open subset $U$ called plots of any $\mathbb{R}^{m}$ into $\tilde{M}$ satisfying the following requirements:

i) If $j: U_{1} \rightarrow U_{2}$ is a smooth map from $U_{1}$ into $U_{2}$ and $\left(\phi_{U_{2}}, U_{2}\right)$ is a plot, $\left(\phi_{U_{2}} \circ j, U_{1}\right)$ is still a plot called the composite plot

ii) The constant map is a plot

iii) If $U_{1}$ and $U_{2}$ are two open subset of the same linear space $\mathbb{R}^{m}$ and if $\left(\phi_{U_{1}}, U_{1}\right)$ and $\left(\phi_{U_{2}}, U_{2}\right)$ are two plots, the union map $\phi_{U_{1} \cup U_{2}}$ realizes a plot from $U_{1} \cup U_{2}$ into $\tilde{M}$.

This allows to Chen and Souriau to define a form. A form $\sigma$ is given by the data of smooth forms $\phi_{U}^{*} \sigma$ associated to each plot $\left(\phi_{U}, U\right)$. The system of forms $\phi_{U}^{*} \sigma$ over $U$ has moreover to satisfy the following requirements: if $\left(\phi_{U_{2}} \circ j, U_{1}\right)$ is a composite plot, $\left(\phi_{U_{2}} \circ j\right)^{*} \sigma$ is equal to $j^{*} \phi_{U_{2}}^{*} \sigma$.

In the theory of Chen and Souriau, the exterior derivative $d \sigma$ of $\sigma$ is given by the data $\mathrm{d} \phi_{U}^{*} \sigma$.

This allows Souriau to define de Rham cohomology of manifolds with singularities.

Definition 3. A stochastic m-dimensional plot $\left(U, \phi_{i}, \Omega_{i}\right)$ of $\Omega_{x, 1 / 2-\epsilon}^{n}(M)$ is given by the following data:

i) $U$ is an open subset of $\mathbb{R}^{m}$

ii) $\Omega_{i}$ is a countable measurable partition of $\Omega$

iii) $u \rightarrow \phi_{i}(u)$.(.) is a smooth application from $U$ into $S P_{\infty}$

iv) $\phi_{i}(u)_{1}(S)$ belongs to $M$ over $\Omega_{i}$ and is equal to $x$ outside a deterministic set $V$ of $\mathbb{R}^{n}$, independent of $\Omega_{i}$.

Let us explain why we do the partition of $\Omega$ in $\Omega_{i}$, i.e., we describe the way how we deform a random $n$-fold loop. For instance, we would like to retract a random $n$-fold loop in a constant loop: it is in general impossible to do that globally. We can do that only locally: this explains the apparition of this partition of $\Omega$ in $\Omega_{i}$. 
In the sequel, we will restrict our stochastic plots to the terminal time $\phi_{i, 1}$, i.e., we identify two elements $S P_{\infty}$ by their terminal time. We call such plots $\left(U, \phi_{i, 1}, \Omega_{i}\right)$. We will give an example of stochastic plots starting from the considerations of [36] to construct Brownian cylinders attached to branes.

Let $F(u, S, y)$ be a smooth function from $U \times \mathbb{R}^{n} \times M$ into $M$ such that outside a compact set of $\mathbb{R}^{n}, F(u, S, y)=x$. Then $u \rightarrow\left\{S \rightarrow F\left(u, S, x_{1}(S)\right)\right\}$ defines a stochastic plot from the open subset $U$ into $\Omega_{x, 1 / 2-\epsilon}^{n}(M)$.

Since we have a stochastic diffeology associated to $\Omega_{x, 1 / 2-\epsilon}^{n}(M)$, we can construct a stochastic cohomology theory associated with this diffeology, according to the previous works of Léandre ([25], [27], [28], [30], [31], [33], [34]).

Definition 4. An element $\sigma_{s t}$ of $\Lambda_{s t}^{k}$, the set of stochastic forms of degree $k$, is given by the following data: let us consider a stochastic $m$-dimensional plot $\phi=$ $\left(U, \phi_{i, 1}, \Omega_{i}\right)$ with values in $\Omega_{x, 1 / 2-\epsilon}^{n}(M)$. On $U$, we associate a random $k$-form $\sigma_{U}=\phi^{*} \sigma_{s t}$. Moreover, the system of random forms $\phi^{*} \sigma_{s t}$ checks the following properties:

i) Let $j$ be a deterministic map from $U^{1}$ into $U^{2}$ and let $\phi^{2}=\left(U^{2}, \phi_{i, 1}^{2}, \Omega_{i}\right)$ be a stochastic plot. We associate with these data the composite plot $\phi^{1}=$ $\left(U^{1}, \phi_{i, 1}^{1}, \Omega_{i}\right)$ given by $\phi_{i, 1}^{1}\left(u_{1}\right)=\phi_{i, 1}^{2}\left(j\left(u_{1}\right)\right)$. We have, almost, surely as random forms

$$
\phi^{1 *} \sigma_{s t}=j^{*} \phi^{2 *} \sigma_{s t}
$$

ii) If $\phi^{1}=\left(U, \phi_{i}^{1}, \Omega_{i}^{1}\right)$ and $\phi^{2}=\left(U, \phi_{i}^{2}, \Omega_{i}^{2}\right)$ are two stochastic plots such that $\phi_{i, 1}^{1}=\phi_{j, 1}^{2} \circ \psi$ on a set of probability not 0 in $\Omega_{j}$ for a given measurable transformation $\psi$ of $\Omega$, then

$$
\phi^{1 *} \sigma_{s t}=\phi^{2 *} \sigma_{s t} \circ \psi
$$

almost surely over this set, where the two plots are corresponding by the transformation $\psi$.

We can define the stochastic exterior derivative of a stochastic form $\sigma_{s t}$ by the data $\mathrm{d} \phi^{*} \sigma_{s t}$ for any stochastic plot $\phi$.

Remark 5. We can identify two stochastic plots $\left(u, \phi_{i, 1}^{1}, \Omega_{i}^{1}\right)$ and $\left(U, \phi_{j, 1}^{2}, \Omega_{j}^{2}\right)$ if $\phi_{i, 1}^{1}()=.\phi_{j, 1}^{2}($.$) almost surely over \Omega_{i}^{1} \cap \Omega_{j}^{2}$ for all $i$ and $j$.

Remark 6. Since $S \rightarrow \phi_{i, 1}(u)(S)$ has a smooth version in $u$ and a $1 / 2-\epsilon$ Hölder version in $S$, a Frechet smooth form over $\Omega_{x, 1 / 2-\epsilon}^{n}(M)$, the $n$-fold $1 / 2-\epsilon$ Hölder loop space, defines a stochastic form. So we have the set of Frechet smooth 
forms $\Lambda_{1 / 2-\epsilon}^{k}$ for the compact support Hölder topology over $\Omega_{x, 1 / 2-\epsilon}^{n}(M)$ and, naturally

$$
\Lambda_{1 / 2-\epsilon}^{k} \subseteq \Lambda_{s t}^{k}
$$

Theorem 7. The set of stochastic forms $\oplus \Lambda_{\text {st }}^{k}=\Lambda_{\text {st }}$ is a differential algebra for the stochastic exterior derivative.

Proof: Let $\phi$ be a stochastic plot. $\sigma_{s t}^{1} \wedge \sigma_{s t}^{2}$ is defined by $\phi^{*} \sigma_{s t}^{1} \wedge \phi^{*} \sigma_{s t}^{2}$ and $\mathrm{d} \sigma_{s t}$ is defined by $\mathrm{d} \phi^{*} \sigma_{s t}$.

Definition 8. The stochastic de Rham cohomology groups $H_{s t}^{k}$ are defined by $\operatorname{Ker}_{\mathrm{d}} \Lambda_{s t}^{k} / \operatorname{Im}_{\mathrm{d}} \Lambda_{s t}^{k-1}$.

Remark 9. Instead of using heat-kernel diffeology, we can use the random field diffeology where we use only the condition

$$
E\left[\left|X(S)-X\left(S^{\prime}\right)\right|^{p}\right]^{1 / p} \leq C_{p}^{1} d\left(S, S^{\prime}\right)^{1 / 2} .
$$

\section{Isomorphism of Cohomology}

Let us make the following hypothesis:

Hypothesis $\mathbf{H}$ : the compact manifold $M$ is $n$-connected.

$\Omega_{x, 1 / 2-\epsilon}^{n}(M)$ is imbedded in $\Omega_{x, 1 / 2-\epsilon}^{n}(M)$, whose differentiable structure is inherited from the differentiable structure of the Hölder $n$-loop space of the linear space $\mathbb{R}^{d} \Omega_{0,1 / 2-\epsilon}^{n}\left(\mathbb{R}^{d}\right)$. In order to obtain an atlas of the manifold $\Omega_{x, 1 / 2-\epsilon}^{n}(M)=$ $\cup \Omega_{x, 1 / 2-\epsilon}^{r, n}(M)$, we proceed as follows: let $I(r)$ be a cube of center 0 and radius r. $\Omega_{0,1 / 2-\epsilon}^{r, n}(M)$ is the set of maps from $\mathbb{R}^{n}$ into $M$ which are equal to 0 outside $I(r)$. A smooth function $F$ over $\Omega_{x, 1 / 2-\epsilon}^{n}(M)$ is a function whose restriction $F^{r}$ to $\Omega_{x, 1 / 2-\epsilon}^{r, n}(M)$ is smooth, for the structure of the manifold of $\Omega_{x, 1 / 2-\epsilon}^{r, n}(M)$ given below. We consider $i_{r}$ to be the inclusion from $\Omega_{x, 1 / 2-\epsilon}^{r, n}(M)$ into $\Omega_{x, 1 / 2-\epsilon}^{r+1, n}(M)$, ( $r$ belongs to $\mathbb{N}$ ). A smooth form $\sigma$ over $\Omega_{x, 1 / 2-\epsilon}^{n}(M)$ is a sequence of smooth forms $\sigma_{r}$ over $\Omega_{x, 1 / 2-\epsilon}^{r, n}(M)$ such that $i_{r}^{*} \sigma_{r+1}=\sigma_{r}$. It remains to define an atlas over $\Omega_{0,1 / 2-\epsilon}^{r, n}(M)$. Let $S_{r}$ be an element of $\mathbb{R}^{n}$ which is far from $I(r)$. There is a family of straight lines $f_{r, S}(t)$ which starts at $S_{r}$ and arrive in $S$ in $I(r)(t \leq 1)$. We write

$$
\gamma(S)=\exp _{\gamma_{\alpha}\left(f_{r, S}(1)\right)}\left[\tau_{S}(1) H(S)\right]
$$


where $\tau_{s}(t)$ is the parallel transport over the curve $t \rightarrow \gamma_{\alpha}\left(f_{r, S}(t)\right)$ and $\gamma_{\alpha}($. is a countable dense family of smooth elements of $\Omega_{x, 1 / 2-\epsilon}^{r, n}(M)$. We choose, for $S \rightarrow H(S)$ an Hölder map from $\mathbb{R}^{n}$ into $\mathbb{R}^{d}$ which equals to 0 outside $I(r)$. Since $M$ is supposed to be $n$-connected, we can deform the element $\gamma_{\alpha}($.$) with support$ in $I(r)$ in the constant element, and we get the deformation $(t, S) \rightarrow \gamma_{\alpha}\left(f_{r, S}(t)\right)$ which is smooth in the two parameters. Therefore, $S \rightarrow \tau_{S}(1)$ is smooth, and so, we can choose, for $S \rightarrow H(S)$ an Hölder map from $\mathbb{R}^{n}$ into $\mathbb{R}^{d}$ which equals to 0 outside $I(r)$. The basis of a neighborhood is given by $O_{r, \alpha, \beta}$ such that the $1 / 2-\epsilon$ norm of $H($.$) is smaller than \beta$.

Let $S^{i, N}$ be the dyadic subdivision of $I(r)$ of length $2^{N}$. Let $x_{i}$ be a finite set of elements of $M$ such that the balls $B\left(x_{i}, \delta\right)$ constitute an open cover of $M$ for $\delta$ small enough. By induction over $n$, we can find a system of elements $\gamma^{r, N}(S)$ of $\Omega_{x, 1 / 2-\epsilon}^{r, n}(M)$ such that for all sites $S^{i, N}, \gamma^{r, N}\left(S^{i, N}\right)$ is equal to one of the $x_{j}$ and the distance between $\gamma^{r, N}\left(S^{i, N}\right)$ and $\gamma\left(S^{i^{\prime}, N}\right)$ for two contiguous sites is smaller than $\delta$. For $n=1$, we choose piecewise geodesics. Moreover

$$
\int_{I(r)}\left|\frac{\partial^{r}}{\partial s_{1} . . \partial s_{n}} \gamma^{r, N}(S)\right|^{2} \mathrm{~d} s<\infty .
$$

Let $O^{r, N}\left(\delta^{\prime}\right)$ be the set of open balls for the uniform norm over $\mathbb{R}^{n}$ of element $\gamma($.$) of \Omega_{x, 1 / 2-\epsilon}^{n}(M)$ such that

$$
\sup _{S \in \mathbb{R}^{n}} d\left(\gamma(S), \gamma^{r, N}(S)\right) \leq \delta^{\prime}
$$

We can find $\delta^{\prime}$ small enough such that the set of $O^{r, N}\left(\delta^{\prime}\right)$ constitutes a cover of $\Omega_{x, 1 / 21-\epsilon}^{n}(M)$.

Remark 10. If we suppose $\gamma(S)=0$ outside $I(r)$, we do not get an open subset of $\Omega_{x, 1 / 2-\epsilon}^{n}(M)$

Theorem 11. Let $O^{r, N}\left(\delta^{\prime}\right)$ be the previous cover of $\Omega_{x, 1 / 2-\epsilon}^{n}(M)$. There exists a smooth partition of unity $g_{r, N}$ associated with this cover for $\Omega_{x, 1 / 2-\epsilon}^{n}(M)$.

Proof: Let us introduce a smooth function $g$ from $[0, \infty]$ into $[1, \infty]$ which is exactly equal to 1 over $\left[0, \delta^{\prime}\right]$. We suppose that $g$ behaves as $\left|\delta^{\prime \prime}-r\right|^{-k}$ when $r \rightarrow \delta^{\prime \prime}$ by lower values and is infinite for $r>\delta^{\prime \prime}$ and finite for $r<\delta^{\prime \prime}$. We introduce a smooth function $h$ from $[1, \infty[$ on $[0,1]$ with compact support which 
is equal to one only at 1 . We introduce the function

$$
F_{r, N}(\gamma)=h\left(\int_{\mathbb{R}^{n}} \frac{g\left(d\left(\gamma(S), \gamma^{r, N}(S)\right) \mathrm{d} S\right.}{\operatorname{vol} I(r)}\right) .
$$

Since we consider the Hölder $n$-fold loop space, we have

$$
\int_{\mathbb{R}^{n}} g\left(d\left(\gamma(S), \gamma^{r, N}(S)\right) \mathrm{d} S=\infty\right.
$$

if $\sup _{\mathbb{R}^{n}} d\left(\gamma(S), \gamma^{r, N}(S)\right) \leq \delta^{\prime \prime}$ for $k$ big enough. This shows that $F_{r, N}(\gamma)$ is smooth for the compact Hölder topology, is equal to one over $B\left(\gamma^{r, N}, \delta^{\prime}\right) \cap$ $\Omega_{x, 1 / 2-\epsilon}^{r, N}(M)$ for the uniform distance, and is equal to zero outside the ball $B\left(\gamma^{r, N}, \delta^{\prime \prime}\right)$ for the uniform distance.

If $\gamma$ belongs to some $B\left(\gamma^{r, N+1}, \delta^{\prime}\right)$ without belonging to any $B\left(\gamma^{r, N^{\prime}}, \delta^{\prime}\right)$ for $N^{\prime} \leq N$ for the balls defined for the uniform distance, we have the property that $\sup _{\left|S-S^{\prime}\right|<2^{-(N+1)}} d\left(\gamma(S), \gamma\left(S^{\prime}\right)\right)>\delta_{1}$ for a conveniently small $\delta_{1}$ over the cube $I(r)$. We regularize the functional $\sup _{\left|S-S^{\prime}\right|<2}-(N+1)$ as in [30]. We put $\psi\left(S, S^{\prime}\right)=d\left(\gamma(S), \gamma\left(S^{\prime}\right)\right)^{2}$ for $\gamma(S)$ and $\gamma\left(S^{\prime}\right)$ close enough and $\psi\left(S, S^{\prime}\right)=1$ for $\gamma(S)$ and $\gamma\left(S^{\prime}\right)$ far. We put

$$
H_{r, N+1}(\gamma)=C(r) 2^{C(N+1)} \int_{I(r) \times I(r),\left|S-S^{\prime}\right|<2^{-(N+1)}} \frac{\mathrm{d} S \mathrm{~d} S^{\prime}}{\left(\delta_{2}-\psi\left(S, S^{\prime}\right)\right)^{+k}}
$$

for some $\delta_{2}<\delta_{1}$ close to $\delta_{1}$. We choose a function $f_{1}$ with support $[0, C]$ from $[0, \infty]$ into $[0,1]$ and we introduce the functional

$$
g_{r, N+1}(\gamma)=1-f_{1}\left(H_{r, N+1}\right)
$$

If $\sup _{S \in I(r), S^{\prime} \in I(r)\left|S-S^{\prime}\right|<2^{-(N+1)}} \geq \delta_{2}, H_{r, N+1}$ is infinite, because we work on the Hölder $n$-fold loop space, if $k$ is big enough. Therefore, $G_{r, N+1}$ is smooth on the Hölder $n$-fold loop space. Moreover, if this condition is checked, $G_{r, N+1}(\gamma)=$ 1. Moreover, $\psi_{r}=\sum_{N} F_{N, r} G_{r, N+1}$ is smooth over the Hölder $n$-fold loop space for the compact support Hölder topology. It is strictly positive over $\Omega_{x, 1 / 2-\epsilon}^{r, n}(M)$. Let us recall that $r$ is an integer. By using a similar construction to that used before, we put

$$
F_{r}(\gamma)=h\left(\int_{I(r+1)-I(r)} \frac{\mathrm{d} S}{\left(\delta_{3}-d(\gamma(S), x)^{2}\right)^{+k}}\right)
$$


$F_{r}(\gamma)=0$ implies that $\gamma$ belongs to some $O^{r+1, N^{\prime}}$ for some $\gamma^{r+1, N^{\prime}}$ where $\gamma^{r+1, N^{\prime}}$ is not equal to $x$ over a site of $I(r+1)$ which is not in $I(r)$. In $\psi_{r}$, we select only the $\gamma^{r, N}$ where there is a site $S$ in $I(r)-I(r-1)$ where $\gamma^{r, N}(S) \neq x$. Therefore, we put

$$
\Psi=\sum F_{r} \psi_{r}
$$

$\Psi$ is smooth over $\Omega_{x, 1 / 2-\epsilon}^{n}(M)$, finite and strictly positive. The partition of unity is given by

$$
g_{r, N}=\frac{F_{N, r} G_{r, N+1} F_{r}}{\Psi} .
$$

The support of $g_{r, N}$ is included in the balls for the uniform distance $B\left(\gamma^{r, N}, \delta^{\prime \prime}\right)$, which constitutes a cover of $\Omega_{x, 1 / 2-\epsilon}^{n}(M)$.

Let us remark that if we put $\alpha=(r, N)$, the system $O_{\alpha}=B\left(\gamma^{r, N}, \delta^{\prime \prime}\right)$ constitutes a cover of the Hölder $n$-fold loop space. Moreover, there is a lexicographic order on the system of indices. First, we distinguish if $r \leq r^{\prime}$ or not. Secondly, we distinguish if $N \leq N^{\prime}$ or not. And as a last step, we use the lexicographic order over the lattice on the cube $I(r)$. In the sequel, $\alpha_{1}<\alpha_{2}<\ldots \alpha_{k}$. $O_{\alpha}$ is contractible if $\delta^{\prime \prime}$ is small enough, as well as $O_{\alpha_{1}, . ., \alpha_{k}}=\cap O_{\alpha_{i}}$, if this intersection is not empty. If it is the case, let us introduce a smooth element $\gamma_{\alpha_{1}, . ., \alpha_{k}}$ of $O_{\alpha_{1}, . ., \alpha_{k}}$ There exists a smooth functional from $O_{\alpha_{1}, . ., \alpha_{k}} \times[0,1]$ over $O_{\alpha_{1}, . ., \alpha_{k}}$ such that

i) $F_{\alpha_{1}, . ., \alpha_{k}}(\gamma(), t).(S)=F_{\alpha_{1}, . ., \alpha_{k}}(S, \gamma(S), t)$

ii) $F_{\alpha_{1}, . ., \alpha_{k}}(., .,$.$) is smooth in S, \gamma(S)$ and $t$

iii) $F_{\alpha_{1}, . ., \alpha_{k}}(S, \gamma(S), 1)=\gamma(S)$

iv) $F_{\alpha_{1}, . ., \alpha_{k}}(S, \gamma(S), 0)=\gamma_{\alpha_{1}, . ., \alpha_{k}}(S)$.

In order to construct this retraction map, we introduce the evaluation map $\xi_{S}$ : $\gamma(.) \rightarrow \gamma(S)$. We remark that $\xi_{S} O_{\alpha_{1}, . ., \alpha_{k}}$ is a convex set. If $\gamma(.) \in O_{\alpha_{1}, . ., \alpha_{k}}, \gamma(S)$ and $\gamma_{\alpha_{1}, . ., \alpha_{k}}(S)$ are joined by a unique geodesic which is included in $\xi_{S} O_{\alpha_{1}, . ., \alpha_{k}}$. We put

$$
F_{\alpha_{1}, . ., \alpha_{k}}(\gamma(.), t)(S)=\exp _{\gamma_{\alpha_{1}, . ., \alpha_{k}}(S)}\left[t\left(\gamma(S)-\gamma_{\alpha_{1}, . ., \alpha_{k}}(S)\right)\right]
$$

in order to construct our retraction application. $\gamma(S)-\gamma_{\alpha_{1}, . ., \alpha_{k}}(S)$ denotes the vector of the unique geodesic joining $\gamma_{\alpha_{1}, . ., \alpha_{k}}(S)$ to $\gamma(S)$ : it is therefore a vector over $\gamma_{\alpha_{1}, . ., \alpha_{k}}(S)$.

Let $O$ be an open subset for the compact Hölder topology on the $n$-fold loop space. It is given by a sequence $O_{r}$ of an open subset of $\Omega_{x, 1 / 2-\epsilon}^{r, n}(M)$ such that $i_{r} O_{r} \subseteq I_{r+1}$. 
Let $\Lambda_{O, s t}^{k}$ be the set of stochastic forms given in a similar set for the loop space in [30].

Definition 12. An element $\sigma_{O, s t}$ of $\Lambda_{O, s t}^{k}$ : Let $\left(U, \phi_{i}, \Omega_{i}\right)$ be a stochastic plot with values in $\Omega_{x, 1 / 2-\epsilon}^{n}(M)$. By definition, it takes its values in some $\Omega_{x, 1 / 2-\epsilon}^{r, n}(M)$ Let $U_{i}$ be the reciprocal image by $\phi_{i, 1}$ of $O^{r}$ over $\Omega_{i}$. It defines a random subset of $U_{i}$. We associate a random smooth form over $U_{i} \phi^{*} \sigma_{O, s t}$, which satisfies a system of consistency relations analogous to Definition 4.

We refer to [30], definition II.5. As an analogue to Lemma II.6 of [30], we have:

Lemma 13. $\Lambda_{O, s t}^{k}$ is a differential algebra for the stochastic exterior derivative.

We have also a lemma analogous to Lemma II.7 in [30]:

Lemma 14. The stochastic de Rham cohomology associated to $\Lambda_{O_{\alpha_{1}, \ldots, \alpha_{k}}, \text { st }}$ is equal to $\mathbb{R}$ in degree 0 and is equal to 0 in others degrees, if $O_{\alpha_{1}, . ., \alpha_{k}} \neq \emptyset$.

The proof is exactly the same as the proof of Lemma II.7 of [30]. It is based upon the fact that if $\phi(u)$.(.) is a plot on $U$, then $F(t, ., \phi(u) .()$.$) is still a plot on$ $[0,1] \times U$ if $F$ is a smooth function in the three argument $(t, S, y)$.

We deduce as in [30], theorem II.9, by using the argument of [9], pp. 166-167, the following lemma:

Lemma 15. The stochastic cohomology of $\Omega_{x, 1 / 2-\epsilon}^{n}(M)$ is equal to the Cech cohomology of the Hölder n-fold loop space associated with the cover $O_{\alpha}$.

The proof is based upon a suitable spectral sequence and the fact that there are partitions of unity associated with the cover $O_{\alpha}$.

We can repeat Lemma 13 and Lemma 14 for the de Rham cohomology group $\Omega_{x, 1 / 2-\epsilon}^{n}(M)$ associated to deterministic smooth forms over it.

For that, we get the following theorem, whose proof is exactly the same as the proof of Theorem A.1 in [30].

Let $F$ be an application from $\mathbb{R}^{n} \times \mathbb{R}^{d}$ into $\mathbb{R}^{d}$ which is smooth and bounded with bounded derivatives of all orders. Let $\Psi$ be the application from $\Omega_{0,1 / 2-\epsilon}^{n}\left(\mathbb{R}^{d}\right)$ into itself defined by

$$
\gamma(.) \rightarrow\{S \rightarrow F(S, \gamma(S))\} .
$$

The application $\Psi$ is called Nemytski map in the literature. We suppose that $F(S, 0)=0$. 
Theorem 16. $\Psi$ is smooth.

This means that $\Psi$ is Fréchet smooth from $\Omega_{0,1 / 2-\epsilon}^{r, n}\left(\mathbb{R}^{d}\right)$ into $\Omega_{0,1 / 2-\epsilon}^{r, n}\left(\mathbb{R}^{d}\right)$ for all integers $r$.

We deduce the following from Theorem 16:

Lemma 17. The deterministic de Rham cohomology of $O_{\alpha_{1}, . ., \alpha_{k}}$ is equal to $\mathbb{R}$ in degree zero and to zero in the others cases, if $O_{\alpha_{1}, . ., \alpha_{k}} \neq \emptyset$.

We deduce also the following lemma, as in [9], pp. 166-167:

Lemma 18. The deterministic de Rham cohomology groups of $\Omega_{x, 1 / 2-\epsilon}^{n}(M)$ are equal to the Cech cohomology groups of $\Omega_{x, 1 / 2-\epsilon}^{n}(M)$ associated to the cover $O_{\alpha}$.

From Lemma 17 and Lemma 18, we deduce the theorem which is the goal of this part:

Theorem 19. The stochastic de Rham cohomology groups of $\Omega_{x, 1 / 2-\epsilon}^{n}(M)$ are equals to the determinist de Rham cohomology groups of $\Omega_{x, 1 / 2-\epsilon}^{n}(M)$.

\section{Little Cube Operad and Stochastic Homology}

Let us recall the definition of an operad. (For all material about operads, we refer to the survey [38]).

Definition 20. A topological operad a is given by the following data:

i) A topological space $a(k), k \in N$ endowed with an action of the symmetric group $S_{k}$.

ii) A continuous action from $a(k) \times a\left(j_{1}\right) \times . . \times a\left(j_{k}\right)$ into $a\left(j_{1}+. . j_{k}\right)$ which is compatible with the different action of the symmetric group.

Example 21. We consider the little cube operad. Let I be the compact subset $[-1,1]$ of $\mathbb{R}$ and $I^{n}=\prod_{i=1}^{n}[-1,1] . F_{n}(k)$ is the space of all maps $\coprod_{i \in[1, k]} d(i)$ : $\coprod_{i \in[1, k]} I^{n} \rightarrow I^{n}$ such that each map $d(i): I^{n} \rightarrow I^{n}$ is a composition of translations and dilatations, and the maps $d(i)$ have disjoint images. This carries a natural action of the symmetric group. The action of $F_{n}(j) \times F_{n}\left(k_{1}\right) \times . . \times F_{n}\left(k_{j}\right)$ is given as follows: let $\left.l \in] k_{1}+. . k_{i} ; k_{1}+. . k_{i+1}\right], l=k_{1}+. . k_{i}+l_{i}, d^{\text {tot }}(l)$ is given as follows. We consider the box given by $d^{k_{i}}\left(l_{r}\right)$ which is included in $I^{n}$, and 
we apply $d(i)$ in $F_{n}(j)$. From this procedure, we obtain a system of $k_{1}+. .+k_{j}$ disjoint boxes, which are compatible with the action of the different symmetric groups.

Definition 22. Let us consider a topological vector space $V$. It is endowed with a structure of a-algebra if there is an action $\rho(k): a(k) \times V^{\otimes k} \rightarrow V\left(V^{\otimes k}\right.$ is the algebraic tensor product) compatible with the different actions of the symmetric group $S_{k}$ and the operation of operads.

Example 23. It is the classical example of algebraic topology. Let us consider $\Omega_{x, 1}^{1, n}(M)$ as the space of $C_{1}$ maps from $I^{n}$ into $M$, such that over a neighborhood of the boundary of $I^{n}$, the map is equal to $\left.x . F_{n}(k)\right)$ acts clearly over $\Omega_{x, 1}^{1, n}(M) \times \ldots \times \Omega_{x, 1}^{1, n}(M)$ where the product is taken $k$ times by the translation and dilatations of the time and concatenation of the spheres which are obtained through this procedure. $F_{n}(k)$ acts over a product of $k$ cycles in $\Omega_{x, 1}^{1, n}(M)$ of dimension $j_{i}$, and we get a cycle of dimension $\sum j_{i}$. We deduce an action of $F_{n}(k)$ over $\otimes H_{j_{i}}\left(\Omega_{x, 1}^{1, n}(M), \mathbb{Z}\right)$ into $H_{\sum j_{i}}\left(\Omega_{x, 1}^{1, n}(M), \mathbb{Z}\right)$ which is clearly compatible with the different actions of the symmetric group (see [40], [8] for more details).

The goal of this part is to produce an analogous statement for the stochastic homology groups of $\Omega_{x, 1 / 2-\epsilon}^{1, n}(M)$. We will slightly change the probabilistic model because we work over $I^{n}$, and we will repeat the considerations of [25] in order to define stochastic homology groups.

Since we work with $\Omega_{x, 1 / 2-\epsilon}^{1, n}(M)$, instead of taking the Hilbert structure (1), we will choose the space of functions from $[-1,1]$ into $\mathbb{R}$ such that

$$
\int_{-1}^{+1}|f(s)|^{2} \mathrm{~d} s+\int_{-1}^{+1}\left|f^{\prime}(s)\right|^{2} \mathrm{~d} s=\langle f, f\rangle<\infty
$$

with Dirichlet boundary conditions $f(1)=f(-1)=0$. With this Hilbert structure, we repeat the considerations of Section 2.

$F_{n}(k)$ clearly acts over $S P_{\infty}^{\otimes k}$ because (8) and (9) are still checked when we perform a translation and a dilatation over $I^{n}$. Therefore, $F_{n}(k)$ acts over the product $\prod_{i=1}^{n}\left(U^{j}, \phi_{i}^{j}, \Omega_{i}^{j}\right)$ of stochastic plots $j=1, . ., k$ and all the requested compatibility conditions are checked. We get a stochastic plot parametrized by $\prod U^{j}$.

We can give the definition of an $m$-dimensional stochastic simplex of the Hölder $n$-fold loop space $\Omega_{x, 1 / 2-\epsilon}^{1, n}(M)$. 
Definition 24. Let $\Delta^{m}$ be the canonical simplex of $\mathbb{R}^{m}$. A stochastic simplex of $\Omega_{x, 1 / 2-\epsilon}^{1, n}(M)$ is given by the restriction of $\Delta^{m}$ to a stochastic plot of a neighborhood $U$ of $\Delta^{m}$ into $\Omega_{x, 1 / 2-\epsilon}^{1, n}(M)$.

Let us recall that, in this definition, we are only interested in $\left(\Delta^{m}, \phi_{i, 1}, \Omega_{i}\right)$, the end point of $\phi_{i}$.

Let $\Omega_{i}^{j}$ be the sets of the measurable partition of $\Omega$ associated with the stochastic $m$-simplex $\left(\Delta^{m}, \phi_{i, 1}^{j}, \Omega_{i}^{j}\right)$. We consider the countable partition of $\Omega \Omega_{i_{1}}^{j_{1}} \cap . . \cap \Omega_{i_{l}}^{j_{l}}$. This allows us to consider a finite sum or a subtraction of $l$ stochastic $m$-simplices, because the partition is still countable. We define the random boundary $\partial$ of an oriented stochastic simplex of dimension $m$ as a sum of oriented $(m-1)$-simplices as usual. We consider the boundary of a sum or a subtraction of oriented stochastic $m$-simplices. If this random boundary is trivial, we say that we have a stochastic cycle. Moreover, $\partial^{2}=0$.

Definition 25. $H_{k, s t}\left(\Omega_{x, 1 / 2-\epsilon}^{1, n}(M), \mathbb{Z}\right)$ is the $k$-dimensional stochastic homology groups of the Hölder $n$-fold loop space $\Omega_{x, 1 / 2-\epsilon}^{1, n}(M)$ with values in $\mathbb{Z}$.

$F_{n}(k)$ applies the product of $k$ random cycles of dimension $j_{j} l=1, . ., k$ into a random cycle of dimension $\sum j_{j}$.

We deduce the following theorem:

Theorem 26. $H_{*, s t}\left(\Omega_{x, 1 / 2-\epsilon}^{1, n}(M), \mathbb{Z}\right) \otimes \mathbb{R}$ is an algebra for the little cube operad.

Remark 27. It can be shown, as in [25], that $H_{*, s t}\left(\Omega_{x, 1 / 2-\epsilon}^{1, n}(M), \mathbb{Z}\right)$ is equal to the space of random variables from $\Omega$ into the discrete group $H_{*}\left(\Omega_{x, 1}^{1, n}(M), \mathbb{Z}\right)$, from which we are able to get an indirect proof of the previous theorem.

\section{Acknowledgements}

We very much appreciate the warm hospitality of the Mathematical Department of the University of Marrakech, where this work was done. 


\section{References}

[1] Airault H. and Malliavin P., Analysis over Loop Groups, Publ. Univ. Paris VI, Paris, 1991.

[2] Albeverio S., Daletskii A. and Kondratiev Y., Stochastic Analysis on Product Manifolds: Dirichlet Operators on Differential Forms, J. Funct. Anal. 176 (2000) 280-316.

[3] Albeverio S., Daletskii A. and Lytvynov Z., Laplace Operator on Differential Forms Over Configuration Spaces, J. Geom. Phys. 37 (2001) 14-46.

[4] Arai A. and Mitoma I., De Rham-Hodge-Kodaira Decomposition in Infinite Dimension, Math. Ann. 291 (1991) 51-73.

[5] Belopolskaya Y. and Daletskii Y., Stochastic Equations and Differential Geometry, Kluwer, Dordrecht, 1990.

[6] Bendikov A. and Léandre R., Regularized Euler-Poincaré Number of the Infinite Dimensional Torus, Infin. Dimens. Anal. Quantum. Probab. Related. Top. 2 (1999) 617-625.

[7] Bismut J., Large Deviations and the Malliavin Calculus, Birkhauser, Basel, 1984.

[8] Boardman J. and Vogt R., Homotopy Invariant Algebraic Structures on Topological Spaces, Lecture. Notes Math. vol. 347, Springer, New York, 1973.

[9] Bott R. and Tu L., Differential Forms in Algebraic Topology, Springer, New York, 1986.

[10] Brzezniak Z. and Elworthy K., Stochastic Differential Equations on Banach Manifolds, Methods Funct. Anal. Top. 6 (2000) 43-84.

[11] Brzezniak Z. and Léandre R., Stochastic Pants over a Riemannian Manifold, Preprint.

[12] Chen K., Iterated Paths Integrals of Differential Forms and Loop Space Homology, Ann. Math. 97 (1973) 213-237.

[13] Cohen F., The Homology of $C_{n+1}$-spaces, $n \geq 0$, In: Lecture Notes Math. vol. 533, Springer, New York, 1976, pp 207-351.

[14] Elworthy K. and Li X., Special Itô Maps and an $L^{2}$ Hodge Ttheory for one Forms on Path Spaces, Preprint.

[15] Fang S. and Franchi J., De Rham-Hodge-Kodaira Operator on Loop Groups, J. Funct. Anal. 148 (1997) 391-407.

[16] Getzler E. and Jones J., Operads, Homotopy Algebra and Iterated Integrals for Double Loop Spaces, Preprint, University of Warwick, 1994. 
[17] Iglésias P., Thesis, Université de Provence, 1985.

[18] Jones J. and Léandre R., $L^{p}$ Chen Forms on Loop Spaces. In: Stochastic Analysis, M. Barlow and N. Bingham (Eds), Cambridge Univ. Press 1991, pp 104-162.

[19] Kuo H., Diffusion and Brownian Motion on Infinite Dimensional Manifolds, Trans. Amer. Math. Soc. 159 (1972) 439-451.

[20] Kusuoka S., De Rham Cohomology of Wiener-Riemannian Manifold, Preprint, 1990.

[21] Léandre R., Stochastic Moore Loop Space, In: Chaos: The Interplay Between Stochastic and Deterministic Behaviour, P. Garbacszewski (Ed), Lecture Notes in Phys. vol. 457, Springer, New York, 1995, pp 479-502.

[22] Léandre, R., Cohomologie de Bismut-Nualart-Pardoux et cohomologie de Hochschild entiere, In: Séminaire de Probabilités XXX in honour of P. Meyer and J. Neveu, J. Azéma, M. Emery and M. Yor (Eds), Lecture Notes in Math. 1626, Springer, New York, 1996, pp 68-100.

[23] Léandre, R., Brownian Cohomology of an Homogeneous Manifold. In: New Trends in Stochastic Analysis, K. Elworthy, S. Kusuoka and I. Shigekawa (Eds), World Scientific, Singapore, 1997, pp 305-347.

[24] Léandre R., Stochastic Cohomology of the Frame Bundle of The Loop Space, J. Non Linear Math. Phys. 5 (1998) 23-41.

[25] Léandre R., Singular Integral Homology of the Stochastic Loop Space, Infin. Dimens. Anal., Quantum. Probab. Relat. Top. 1 (1998) 17-31.

[26] Léandre R., Cover of the Brownian Bridge and Stochastic Symplectic Action, Rev. Math. Phys. 12 (2000) 91-137.

[27] Léandre R., Anticipative Chen-Souriau Cohomology and Hochschild Cohomology. In: Conférence Moshé Flato II, G. Dito and D. Sternheimer (Eds) Math. Physics. Stud. 22, Kluwer, Dordrecht, 2000, pp 185-199.

[28] Léandre R., A Sheaf Theoretical Approach to Stochastic Cohomology. In: "XXXI Symposium of Mathematical Physics of Torun", R. Mrugala (Ed), Rep. Math. Phys. 46 (2000) 157-164.

[29] Léandre R., Stochastic Adams Theorem for a General Compact Manifold, Rev. Math. Phys. 13 (2001) 1095-1133.

[30] Léandre R., Stochastic Cohomology of Chen-Souriau and Line Bundle over the Brownian Bridge. Probab. Theory Related Fields 120 (2001) 168-182.

[31] Léandre R., Stochastic Diffeology and Homotopy. In: Stochastic Analysis and Mathematical Physics, A. Cruzeiro and J.-C. Zambrini (Eds), Birkhauser, Basel, 2001, pp 51-57. 
[32] Léandre R., Analysis over Loop Space and Topology, Math. Notes. 72 (2002) 212-229.

[33] Léandre R., Stochastic Wess-Zumino-Novikov-Witten Model on the Sphere, Preprint.

[34] Léandre R., Stochastic Wess-Zumino-Novikov-Witten Model on the Torus, J. Math. Phys. 44 (2003) 5530-5568.

[35] Léandre R., Brownian Pants and Deligne Cohomology, J. Math. Phys. 46 (2005) 033503.

[36] Léandre R., Brownian Cylinders and Intersecting Branes, Rep. Math. Phys. 52 (2003) 363-372.

[37] Léandre R. and Smolyanov O., Stochastic Homology of the Loop Space. In: Analysis on Infinite Dimensional Lie Groups and Algebras, H. Heyer and J. Marion (Eds), World Scientific, Singapore, 1999, pp 229-235.

[38] Loday J., La renaissance des opérades, Séminaire Bourbaki 791, Astérisque 237 (1996) 47-75.

[39] Mac Lane S., Categories for the Working Mmathematician, Springer, New York, 1971.

[40] May J., The Geometry of Iterated Loop Spaces., Lecture Notes in Math. 271, Springer, New York, 1972.

[41] Meyer P., Flot d'une équation différentielle stochastique. In: Séminaire de Probabilités XV, J. Azéma, P. Meyer and M. Yor (Eds), Lecture Notes in Math. vol. 850, Springer, New York, 1981, pp 100-117.

[42] Ramer R., On the de Rham Complex of Finite Codimensional Forms on Infinite Dimensional Manifolds, Thesis, Warwick University, 1974.

[43] Shigekawa I., De Rham-Hodge-Kodaira's Decomposition on an Abstract Wiener Space, J. Math. Kyoto. Univ. 26 (1986) 191-202.

[44] Smolyanov O., De Rham Current's and Stoke's Formula in a Hilbert Space, Soviet. Math. Dokl. 33 (1986) 140-144.

[45] Souriau J.-M., Un algorithme générateur de structures quantiques. In: Elie Cartan et les Mathématiques D'aujourd'hui, Astérisque 985 341-399.

[46] Stasheff J., Homotopy Associativity of H-spaces I\&II, Trans. Amer. Math. Soc. 108 (1963) 275-312.

Rémi Léandre

Institut de Mathématiques de Bourgogne

Université de Bourgogne

21078 Dijon, FRANCE

E-mail address: Remi. Leandre@u-bourgogne.fr 